\title{
Postpartum Pancreatic Encephalopathy -A Rare Case
}

\author{
Lekhra OP ${ }^{1}$, Maheshwari $\mathrm{A}^{2}$, Rathore $\mathrm{Y}^{3}$, Athale $\mathrm{S}^{4}$. \\ ${ }^{1}$ Associate Professor, ${ }^{2}$ Sr Resident,${ }^{3}$ Sr.Physiotherapist,${ }^{4}$ Professor\&Head Department of Neurology \\ Sri Aurobindo Medical College and PG Institute,Indore India
}

\begin{abstract}
A young female of postpartum pancreatic encephalopathy is described who presented with convulsions and coma. She had deranged lipase levels in blood, and cerebrospinal fluid along with abnormal white matter signals in MRI brain. As the patient improved with medical management, deranged blood lipase levels and abnormal MRI signals also improved. Pancreatic encephalopathy is a rare complication of pancreatitis in the setting of postpartum period. In any atypical neuropsychiatric condition with abdominal symptoms the possibility of pancreatic encephalopathy should be considered after certain exclusions.
\end{abstract}

Key Words : encephalopathy,metabolic, pancreatic, postpartum

\section{Introduction}

A wide variety of neurological conditions can affect women during pregnancy and puerperium. Common neurological disorders seen in relation to pregnancy and post partum period are eclampsia,venous stroke, epilepsy, demyelination and metabolic encephalopathy ${ }^{1}$. We describe a rare case of postpartum pancreatic encephalopathy in a young female .

\section{Case Report}

A 22 year female developed fever, abdominal pain ,altered sensorium, generalized tonic-clonic seizures and quadriparesis on fifth post partum day.She had an uneventful full term normal delivery.. On examination she had a temperature of (100F). BP 110/70 mmHg, Pulse.100/min. She was in altered sensorium with eye opening on deep painful stimuli. Pupils were bilaterally equal and reacting to light. Eye movements were full. Bilateral fundi were normal .No meningeal signs. Tone increased in all limbs. DTRs - brisk, Motor power grade $3 / 5$ in upper limbs and grade $2 / 5$ in lower limbs. Pin prick sensations were intact. Plantars were mute on either side. Abdomen was distended with normal bowel sounds. MRI Brain showed bilateral symmetrical hyper intense signals on diffusion and T2 FLAIR sequences with low ADC values in posterior limb of internal capsule, posterior centrum semiovale and splenium of corpus callosum suggestive of nonspecific metabolic encephalopathy/demyelination/ischemic injury (Fig1). MR angiogram and venogram did not show any vascular abnormality. EEG revealed diffuse slowing of background activity, with theta waves recorded over all the regions of the scalp.

A set of investigations was done which showed $\mathrm{Hb}-15.2 \mathrm{gm} \%$,TLC-16400,N87\%, L11\%,platelet count 1.35lac,SGPT-35 I units, SerumNa+145meq/l,SerumK+4.6meq/l, Cl-125meq/l, PT-INR 1.3, Blood Urea $59 \mathrm{mg} / \mathrm{dl}$, Serum Creatinine- $0.85 \mathrm{mg} / \mathrm{dl}$.Malaria antigen negative, D-dimer- 230 microgm $/ \mathrm{L}$,Serum Amylase was $340 \mathrm{U} / \mathrm{L}(23-85 \mathrm{U} / \mathrm{L}$ normal) and Serum Lipase was $3931 \mathrm{U} / \mathrm{L}$ (0-160 U/L normal).

In view of her high amylase and lipase and abdominal distention, a CECT abdomen was ordered which showed minimal peripancretic fat stranding and small amount of peripancreatic fluid but no evidence of any hepatobilliary disease. A presumptive diagnosis of acute pancreatitis was considered. A CSF study was done which showed an opening pressure of $15 \mathrm{~cm}$, glucose $59 \mathrm{mg} / \mathrm{dl}$ (corresponding blood sugar $83 \mathrm{mg} / \mathrm{dl}$ ),total cells -2 all lymphocytes, Pandy's test- negative, protein 46mg/dl, VDRL negative with CSF lipase $48 \mathrm{mg} / \mathrm{dl}$.

Patient was managed with intravenous fluids, broad spectrum antibiotics and nutritional support along with physiotherapy. With regular rehabilitation and medical management she gradually regained motor power and improvement in sensorium.Two weeks after onset of illness patient became conscious, oriented, following commands. Her speech had become normal, she was able to walk with partial support and her upper limb power has improved to grade 4/5. Her swallowing reflexes recovered gradually and she accepted feeds orally.A repeat MRI Brain was done which showed far less degree of abnormal signals in the same distribution as compared to previous MRI with no contrast enhancement. A repeat serum Amylase and lipase was done, which were $155 \mathrm{U} / \mathrm{L}$ and $102 \mathrm{U} / \mathrm{L}$ respectively. A final diagnosis of postpartum pancreatic encephalopathy was made 


\section{Figure}

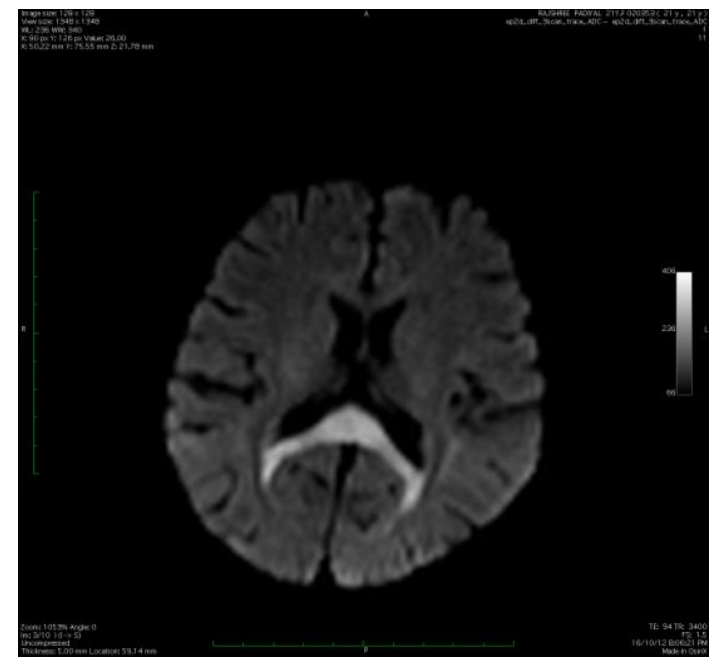

Fig -1 MRI DWI brain shows bilateral symmetrical hyperintense signals on posterior limb of internal capsule,posterior centrum semiovale and splenium of corpus callosum

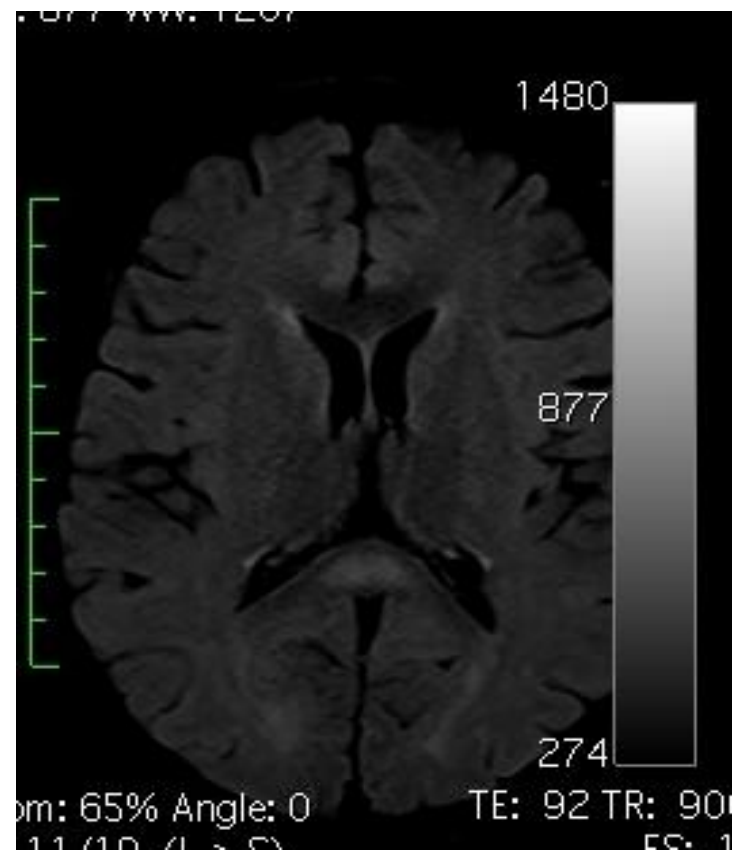

Fig 2- Repeat MRI brain shows far less degree of the abnormal signals in the same areas as compared to Fig1

\section{Discussion}

Epilepsy, cortical vein thrombosis and hepatic encephalopathy have emerged as the predominant neurological disorders in pregnancy and puerperium ${ }^{1}$. In our case striking metabolic abnormalities were disturbed pancreatic function tests as evident by abnormally high serum amylase and serum lipase levels at presentation. Moreover there was a comparable relationship between the appearance of symptoms of encephalopathy, electroencephalographic changes, radiological changes, and the lipase both in blood and CSF. The deranged blood parameters showed resolution as the patient condition improved over two weeks. Hence we considered it as a case of pancreatic encephalopathy. Pancreatic encephalopathy is well described in literature but is quite uncommon in post partum situation ${ }^{2}$.

Pancreatic encephalopathy (PE) is an uncommon complication of acute pancreatitis.It was first described in 1941 by Rothermich and Von Haam ${ }^{3}$.Pancreatic encephalopathy can arise abruptly with altered sensorium and convulsions in the setting of pancreatitis. Neurological signs and symptoms may occur during the first two weeks of acute pancreatitis ${ }^{2,4}$. It can have a gradual onset with behavioral changes, psychomotor 
agitation, disorientation to time, space and person, and visual and auditory hallucinations. It is a diagnosis of exclusion as many situations can mimic this condition, such as electrolyte imbalance, hypo/hyperglycemia, diabetic acidosis, hypo/ hyper-calcaemia, hyperrosmolarity syndrome, renal or hepatic failure, cerebral vascular insufficiency and delirium tremens, ${ }^{5,8,7}$ The pancreatic encephalopathy does not affect the course of the pancreatitis. The recovery usually is uneventful, as in our case ${ }^{5}$

In our case all these conditions of ischaemia, electrolyte imbalance and demyelination were ruled out. Most accepted hypothesis for the pathogenesis of pancreatic encephalopathy mentions lipase as the crucial substance ${ }^{6}$. Pancreatic enzymes (lipase) in the blood stream alters the blood-brain barrier and damage scattered areas throughout the cerebrum, brainstem and cerebellum, inducing increased vascular permeability. The intravascular osmotic pressure decreases and the brain becomes more vulnerable to transudation, and finally results in brain oedema ${ }^{6}$. These areas are picked as patchy white matter signal abnormalities in MRI as described in our patient.. Therefore in any patient with atypical neuropsychiatric condition especially associated with abdominal symptoms and deranged pancreatic parameters along with abnormal white matter abnormalities, pancreatic encephalopathy should be considered after exclusion of infective, ischemic and other common metabolic factors.

\section{References}

[1]. Gupta S, Rohatgi A, Sharma SK,Gurtoo A. A study of neurological disorders during pregnancy and puerperium. Ann Indian Acad Neurol 2006; 9:152-7

[2]. Estrada RV, Moreno J, Martinezet E et al . Pancreatic encephalopathy. Acta Neurol Scand.1979 59:135-139

[3]. Rothermich NO, Von Haam E. Pancreatic encephalopathy. J Clin Endocrinol 1941;1:872-873.

[4]. Sharf B, Bental E. Pancreatic encephalopathy.J Neurol Neurosurg Psychiatry 1971;34:(3) 357-361

[5]. Pallis CA,Lewis PD 1974 The neurology of gastrointestinal disease.Saunders,London

[6]. Ruggieri RM,Lupo L, Piccoli F.Pancreatic encephalopathy : a 7-year follow-up case report and review of the literature . Neuro Sci 2002; ((23):203-205

[7]. Boon P, de Reuck J, Achten E, de Bleeker J. Pancreatic encephalopathy.A case report and review of the literature. Clin Neurol Neurosurg. 1991;93(2):137-41

[8]. Guo-Hui Sun,Yun-Sheng Yang,Qing-Sen Liu,Liu-Fang Cheng ,Xu-Sheng Huang .pancreatic encephalopathy and Wernicke encephalopathy in association with acute pancreatitis:A clinical study. World J of gastroenterology 2006 July 14;12(26):4224-4227 